\title{
Hawking radiation in different coordinate settings: Complex paths approach
}

\author{
S. Shankaranarayanan, T. Padmanabhan and K. Srinivasan \\ IUCAA, Post Bag 4, Ganeshkhind, Pune 411 007, INDIA. \\ E-mail: shanki@iucaa.ernet.in,paddy@iucaa.ernet.in
}

\begin{abstract}
We apply the technique of complex paths to obtain Hawking radiation in different coordinate representations of the Schwarzschild space-time. The coordinate representations we consider do not possess a singularity at the horizon unlike the standard Schwarzschild coordinate. However, the event horizon manifests itself as a singularity in the expression for the semiclassical action. This singularity is regularized by using the method of complex paths and we find that Hawking radiation is recovered in these coordinates indicating the covariance of Hawking radiation as far as these coordinates are concerned.
\end{abstract}

\section{Introduction}

Quantum field theory(QFT) requires the notion of a time-like killing vector field to define particles. In Minkowski space-time existence of a global time-like killing vector, invariant under Lorentz transformation, allows us to identify a preferred vacuum state for the theory. Even in flat space-time, when curvilinear coordinate transformations are allowed there arises other possible killing vector fields which are time-like in part of the manifold. Particle states - in particular vacuum state - can be defined using these killing vector fields. In general, these definitions will not be equivalent and the Minkowski vacuum will appear to be a many particle state according to the new definition. For example, the vacuum state of the Minkowski observer appears to be a thermal state in the uniformly accelerated frame with a temperature given by $T=g / 2 \pi$ ( $g$ is the constant acceleration).

In a general curved space-time, we may not have any time-like killing vector field and hence it is not always possible to identify a preferred set of positive frequency modes. In static space-times, which have a time-like killing vector, it is possible to define positive frequency modes. However in these space-times, there could arise more than one time-like killing vector field making the quantization in different coordinates (describing the same gravitational background) inequivalent. Hence, the concept of a particle is not generally covariant in curved space-times.

A spectacular prediction of quantum field theory in curved space-time is the Hawking radiation from a black hole. Hawking 2] showed that QFT in the background of a body collapsing to a black hole, of mass $M$, will lead, at late times, to a radiation of particles in all modes of the quantum field, with characteristic thermal spectrum at a temperature equal to $(1 / 8 \pi M)$. It is generally believed that the pair production occurring near the horizon in the case of black holes is "real" with the mass of the black hole being converted into energy of the emitted particles. 
But as mentioned in the earlier paragraphs, the concept of a particle in quantum field theory is not generally covariant and depends on the coordinates chosen to describe the particular space-time. It is therefore of interest to study the Hawking effect in other coordinate representations. The original method used by Hawking is closely tied to the standard Schwarzschild coordinate. Hawking's calculation of the particle creation amplitudes (and Bogolubov coefficients) requires the knowledge of the wave modes of the quantum field in the standard Schwarzschild coordinate. The solutions of the wave equation in the Schwarzschild coordinate system cannot be written down in terms of simple functions and hence, the calculation of Bogolubov coefficients to identify the spectrum of radiation becomes intractable. Hence, it is necessary to have a method which does not use wave modes to calculate the emission spectrum.

Hartle and Hawking [3] obtained particle production in the standard black hole space-times using a semiclassical analysis which does not require the knowledge of the wave modes. In this method, the semiclassical propagator for a scalar field propagating in the maximally extended Kruskal manifold is analytically continued in the time variable $t$ to complex values. This analytic continuation gives the result that the probability of emission of particles from the past horizon is not the same as the probability of absorption into the future horizon. The ratio between these probabilities is of the form

$$
P[\text { emission }]=P[\text { absorption }] e^{-\beta E},
$$

where $E$ is the energy of the particles and $\beta^{-1}=(1 / 8 \pi M)$ is the standard Hawking temperature. The above relation is interpreted to be equivalent to a thermal distribution of particles in analogy with that observed in any system interacting with black body radiation. In this analysis, the probability amplitude for the emission/absorption is calculated by identifying a particular path for both these processes in the fully extended Kruskal manifold. Unfortunately, the Kruskal extension plays a vital role in obtaining the thermal spectrum in this analysis, and hence, it cannot be adapted to other coordinate systems.

In Ref. [I], the authors have obtained Hawking radiation without using the Kruskal extension. They have shown that the coordinate singularity present at the horizon, in the standard Schwarzschild coordinate, manifests itself as a singularity in the expression for the classical action $S_{0}$ which occurs in the semiclassical propagator $K\left(r_{2}, t_{2} ; r_{1}, t_{1}\right)$, which is given by

$$
K\left(r_{2}, t_{2} ; r_{1}, t_{1}\right)=N \exp \left(\frac{i}{\hbar} S_{0}\left(r_{2}, t_{2} ; r_{1}, t_{1}\right)\right) .
$$

$S_{0}$ is the action functional satisfying the classical Hamilton Jacobi (HJ) equation for a massless particle to propagate from $\left(t_{1}, r_{1}\right)$ to $\left(t_{2}, r_{2}\right)$ and $N$ is the suitable normalization constant. The authors used the method of complex paths discussed in Ref. [5] where it is used to describe tunneling processes in non-relativistic semiclassical quantum mechanics. The method of complex paths used in ordinary quantum mechanics was modified appropriately to produce a prescription that regularizes the singularity in the action functional and Hawking radiation was recovered as a consequence. This method also provides a heuristic picture of Hawking radiation as tunneling. In this method, by construction, the in modes of the field is in the vacuum state and the tunneling process near the horizon produces a steady out-flux of radiation. The authors consider Unruh vacuum to define particles at future infinity. (See for example, Refs. [6, 7]). 
In this work, we apply the method of complex paths to two non-static coordinate systems of the Schwarzschild space-time and obtain Hawking radiation. The two coordinate systems we consider are: Lemaitre coordinate (a time dependent system) and Painlevé coordinate (a stationary — but not a static — system). The Lemaitre coordinate being a time dependent system, suggests that there could be a genuine particle production. Hence, it is interesting to know what kind of particle spectrum is seen in this coordinate system. In the case of Painlevé coordinate, a priori it not clear whether there exists a genuine particle production!

Also there has been a growing interest in the semiclassical treatment of Hawking radiation in the Painlevé metric (see Refs. [9, 10]) in relation with the hydrodynamic analog models of event horizon. The hydrodynamic analog of an event horizon was suggested by Unruh [11] as a more accessible phenomenon which might shed some light on the Hawking effect and, in particular, on the role of ultra-high frequencies [12]. An event horizon for the sound waves appears in principle wherever there is a closed surface through which a fluid flows inwards at the speed of sound, the flow being subsonic on one side of the surface and supersonic on the other. There is a close analogy between sound propagation on a background hydrodynamic flow, and field propagation on a background in a curved space-time. Determining whether and how sonic black holes radiate sound, in a full calculation beyond the hydrodynamic approximation or in an actual experiment, can thus offer some suggestions about black-hole radiance and its sensitivity to ultra-high frequency physics. It is seen that for an irrotational fluid with constant density and sound speed with the fluid velocity $\mathbf{v}=\sqrt{2 G m / r}$, then one can obtain a metric element which is conformal to Painlevé form of the Schwarzschild space-time. The acoustic horizon of this kind are of interest in understanding the role of ultra-high frequencies in the Hawking radiation.

In these two coordinate representations, Painlevé and Lemaitre, metric has no coordinate singularity at the horizon. We notice that the action function for a classical particle in both these coordinates acquires a singularity at the horizon. In Ref. 胉, it was shown that it is only the singularities that appear in the action that contribute to particle production. For the Lemaitre coordinate, the regularization of the singularity in action is achieved and the Hawking radiation is recovered without reference to the time dependent nature of the system. The same feature is seen even in the stationary system where the singularity represented by the horizon appears as a pole in the expression for the action and upon regularization by a suitable prescription gives Hawking radiation. The temperature associated with the radiation for both the coordinate representations is same as that obtained in the standard Schwarzschild coordinates. We would like to note the following: Even-though the action satisfies a generally covariant HJ equation, when one transforms coordinates, the action integral develops poles and this need to be interpreted. In the case of Kruskal coordinate, which is the maximal extension of Schwarzschild space-time, it is easy to show that the semiclassical action when expressed in terms of Kruskal coordinates does not contain the singularity. [The HJ equation (of a massless particle) when expressed in terms of the Kruskal coordinates $(V, U, \theta, \phi)$ is of the form $\left(\partial S_{0} / \partial V\right)^{2}-\left(\partial S_{0} / \partial U\right)^{2}=0$ (for $\mathrm{S}$-wave i.e $l=0)$. The solution of the equation can be easily obtained and is given by $\left.S_{0}\left(V_{2}, U_{2} ; V_{1}, U_{1}\right)=S_{0}(2,1)=-p_{V}\left(V_{2}-V_{1}\right) \pm p_{U}\left(U_{2}-U_{1}\right).\right]$

To our knowledge, the semi-classical treatment of Hawking radiation for the Lemaitre coordinate has not been performed in the literature. The semi-classical treatment of Hawking radiation for the Painlevé metric is obtained recently by Parikh and Wilczek [9]. The authors considered Hawking radiation as a pair creation outside 
the horizon, with the negative energy particle tunneling into the black hole. They have not considered the contribution of the tunneling of a particle from inside the horizon to outside the horizon to describe the Hawking radiation. Our approach is quite different and could be of more general validity.

Before proceeding to the technical aspects, it is necessary to outline certain conceptual issues regarding the emission spectrum from the black hole in different coordinate systems. The wave modes obtained using semiclassical techniques, in general, are the exact modes of the quantum system in the asymptotic regions. Thus, if the asymptotic structure of the space-time is different for any two coordinates, then the semiclassical wave modes associated with these two coordinate systems will themselves be different. Hence, the spectrum of the radiation emitted, if any, in these two coordinates, with different asymptotic structure, will be completely different. The Lemaitre coordinate can be modeled as that natural to a freely falling observer whose velocity at radial infinity is zero [for more discussion see Sec. (3.2)]. In the asymptotic past and future, the structure of these two coordinate systems are similar; suggesting that the asymptotic wave modes must be the same. In Sec. (价, we have shown that the spectrum of the particles emitted in the two coordinates, Painlevé and Lemaitre, is a thermal spectrum - same as in standard Schwarzschild.

Even though, the two coordinate systems, Painlevé and Lemaitre, possess no singularities at the horizon, the action function for a classical particle acquires singularity at the horizon. The results of Ref. [4] show that the contribution to the thermal radiation of the emitted particles is from the singularities. Thus, it suggests that the temperature associated with the thermal spectrum in these two coordinate systems, Painlevé and Lemaitre, should be the same as that of the standard Hawking temperature. The metric corresponding to the standard Schwarzschild coordinate is time reversal invariant, $t \rightarrow-t$. However, the metric corresponding to the two coordinates, Painlevé and Lemaitre, are not time reversal invariant. The time reversal transformation in these cases correspond to a different physical situation. However, the line elements corresponding to these different physical situations are related to the standard Schwarzschild by a coordinate transformation [see Sec. (3) for more details].

The space-time structure of these two coordinates, Lemaitre and Painlevé, are best analyzed using $\mathrm{R}$ and $\mathrm{T}$ regions introduced by Novikov 16]. The analysis of the space-time structure of the two coordinates, Painlevé and Lemaitre, using $\mathrm{R}$ and $\mathrm{T}$ regions provides an elegant method in understanding the global structure of the space-time, thus making the detailed analysis of particle trajectories unnecessary. Using this analysis, we show that the part/whole of the Lemaitre/Painlevé manifold is doubly mapped w.r.t the standard Schwarzschild space-time. We find that the complex paths we use in our analysis, takes into account of all possible paths satisfying the semiclassical ansatz - irrespective of he multiple mapping of the part/whole of the space-time. The above statement can be better appreciated in the language of pathintegrals.

The Feynman propagator $G_{F}(x, y \mid g)$ for a scalar field, of mass $m$, propagating in a background metric $g_{i k}$ can be written in two equivalent forms as

$$
\begin{aligned}
G_{F}(x, y \mid g) & =\sum_{\text {paths }} \exp (-m \mathcal{R}(x, y)) \\
& =\int_{0}^{\infty} d \tau \exp \left(-m^{2} \tau\right) \int \mathcal{D} x \exp \left(-\frac{1}{4} \int_{0}^{\tau} g_{i k} \dot{x}^{i} \dot{x}^{k} d \eta\right)
\end{aligned}
$$

where $\mathcal{D} x$ is the measure of the path. In the first form, $\mathcal{R}(x, y)$ is the proper length of 
a path connecting the events $x$ and $y$, calculated with the background metric $g_{i k}$ and the sum is over all paths. It is also possible to show that the result is equivalent to the second expression. In the case of space-times where there are no multiple mappings then the measure (of all sets) of paths will be the same. However, in the space-times where part/whole of the manifold is multiply mapped, the measure of certain sets of paths will be different from certain other sets of paths. Hence, it is necessary to do a global analysis of the background space-time to identify the measure of the paths. Similarly, in the method of complex paths, the family of paths we use to calculate the emission and absorption probability takes into account of all paths irrespective of the multiple mapping of the part/whole of the space-time. These facts will have bearing on the interpretation of temperature as explained in Sec. (4).

Our result is of physical significance for the following reason: it is normally assumed that the evaporation process results from an instability of the vacuum in the presence of background metric. The particles are produced at a constant rate suggesting that the Hawking radiation converts the mass of the black hole into energy, thereby decreasing the mass. The decrease in the black hole mass is a physical effect and should be independent of the coordinate transformations and hence Hawking radiation should be covariant. Here we show that this is indeed true by the method of complex paths as far as these coordinates are concerned.

Before concluding this section, we would like to stress the following point: There is no correspondence between the particles detected by a detector (which is coupled to a quantum field $\phi$ ) and the particle spectrum obtained by the field theoretic analysis. This result is known in the context of flat space-times. For example, in the case of uniformly rotating detector, the expectation value of the rotational number operator in the Minkowski space-time is zero whereas the response of a uniformly rotating UnruhDewitt detector is non-zero (For more examples see, Refs. [1, 19, 14, 15]). In the case of classical gravitational backgrounds, defining particles using the particle detectors is non-trivial. The trouble in defining the particle detectors is that the inertial detectors become free falling detectors and in general no two free falling observers will agree on a choice of vacuum. Only in exceptional cases in high symmetry will a set of detector exist that all register no particles, and this set may not even be free falling. These facts will have important implications in comparing the results of a freely falling detector in the Schwarzschild space-time [18] to that of the particle spectrum we obtain for the Lemaitre coordinates in Sec. (4.1).

The organization of the paper is as follows. In Sec. (21), the method of complex paths, as applied to Schwarzschild like space-times, is briefly recapitulated. In Sec. (3), we briefly describe the definition of $\mathrm{R}$ and $\mathrm{T}$ regions of the spherically symmetric space-times and also discuss the properties of the two coordinates which is of our interest, namely Lemaitre coordinate and Painlevé metric. In Sec. (四), we apply the method of complex paths to the two coordinate systems and obtain the temperature corresponding to the radiation. Finally in Sec. (5) we summarize the results of this paper.

Through out this paper, the metric signature we shall adopt is (+---) and the quantum field is a scalar field.

\section{Hawking radiation in Schwarzschild coordinate}

In this section, we briefly describe the recovery of Hawking radiation in the Schwarzschild coordinates using the method of complex paths. We do this in $(1+1)$ 
dimensions since all the physics is contained in the $(t, r)$ plane. The generalization to a massive field and to $(1+3)$ dimensions is straight forward. Consider a certain patch of space-time in $(1+1)$ dimensions, which - in a suitable coordinate system - has the line element, (with $c=1$ )

$$
d s^{2}=B(r) d t^{2}-B^{-1}(r) d r^{2}
$$

where $B(r)$ is an arbitrary function of $r$. We assume that the function $B(r)$ vanishes at some $r=r_{0}$ with $B^{\prime}(r)=d B / d r$ being finite and nonzero at $r_{0}$. The point $r=r_{0}$ indicates the presence of a horizon. It can be easily verified that no physical singularity exists at the horizon since the curvature invariants do not have a singularity on the horizon. Therefore, near the horizon, we expand $B(r)$ as

$$
B(r)=B^{\prime}\left(r_{0}\right)\left(r-r_{0}\right)+\mathcal{O}\left[\left(r-r_{0}\right)^{2}\right]=R\left(r_{0}\right)\left(r-r_{0}\right) .
$$

where it is assumed that $R\left(r_{0}\right) \neq 0$.

The equation satisfied by the minimally coupled massless scalar field in the above background metric is,

$$
\frac{1}{B(r)} \frac{\partial^{2} \Phi}{\partial t^{2}}-\frac{\partial}{\partial r}\left(B(r) \frac{\partial \Phi}{\partial r}\right)=0
$$

The semiclassical wave functions satisfying the above equation are obtained by making the standard ansatz for $\Phi$ which is,

$$
\Phi(r, t)=\exp \left[\frac{i}{\hbar} S(r, t)\right]
$$

where $S$ is a function which is expanded in powers of $\hbar$ of the form

$$
S(r, t)=S_{0}(r, t)+\hbar S_{1}(r, t)+\hbar^{2} S_{2}(r, t) \ldots
$$

Substituting into the wave equation (6) and neglecting terms of order $\hbar$ and greater, we find to the lowest order,

$$
\frac{1}{B(r)}\left(\frac{\partial S_{0}}{\partial t}\right)^{2}-B(r)\left(\frac{\partial S_{0}}{\partial r}\right)^{2}=0 .
$$

Eq. (9) is just the Hamilton-Jacobi equation satisfied by a massless particle moving in the space-time determined by the line element (位). The solution to the above equation is

$$
S_{0}\left(r_{2}, t_{2} ; r_{1}, t_{1}\right)=S_{0}(2,1)=-E\left(t_{2}-t_{1}\right) \pm E \int_{r_{1}}^{r_{2}} \frac{d r}{B(r)} .
$$

The semiclassical kernel $K\left(r_{2}, t_{2} ; r_{1}, t_{1}\right)$ for the particle to propagate from $\left(t_{1}, r_{1}\right)$ to $\left(t_{2}, r_{2}\right)$ in the saddle point approximation can be written down immediately as.

$$
K\left(r_{2}, t_{2} ; r_{1}, t_{1}\right)=N \exp \left(\frac{i}{\hbar} S_{0}\left(r_{2}, t_{2} ; r_{1}, t_{1}\right)\right),
$$

where $S_{0}$ is the action functional satisfying the classical Hamilton-Jacobi equation in the massless limit and $N$ is a suitable normalization constant.

The sign ambiguity (of the square root) in Eq. (10) is related to the "outgoing" $\left(p_{r}=\partial S_{0} / \partial r>0\right)$ or "in-going" $\left(\partial S_{0} / \partial r<0\right)$ nature of the particle. [The momentum of the particle moving in $r$ is given by $p_{r}=\partial S_{0} / \partial r$. Hence, $p_{r}>0$ corresponds to outgoing particle w.r.t the horizon.] As long as points 1 and 2, between which the transition amplitude is calculated, are on the same side of the horizon (i.e. both are in the region $r>r_{0}$ or in the region $r<r_{0}$ ), the integral in the action is well 
defined and real. But if the points are located on opposite sides of the horizon then the integral does not exist due to the divergence of $B^{-1}(r)$ at $r=r_{0}$.

Therefore, in order to obtain the probability amplitude for crossing the horizon we have to give an extra prescription for evaluating the integral [13. Since the horizon defined by $B\left(r_{0}\right)=0$ is null we may carry out the calculation in Euclidean space or - equivalently - use an appropriate $i \epsilon$ prescription to specify the complex contour over which the integral has to be performed around $r=r_{0}$. The prescription we use is that we should take the contour for defining the integral to be an infinitesimal semi-circle above the pole at $r=r_{0}$ for outgoing particles on the left of the horizon and ingoing particles on the right. Similarly, for in-going particles on the left and outgoing particles on the right of the horizon (which corresponds to a time reversed situation of the previous cases) the contour should be an an infinitesimal semi-circle below the pole at $r=r_{0}$. Equivalently, this amounts to pushing the singularity at $r=r_{0}$ to $r=r_{0} \mp i \epsilon$ where the upper sign should be chosen for outgoing particles on the left and in-going particles on the right while the lower sign should be chosen for in-going particles on the left and outgoing particles on the right. For the Schwarzschild case, this amounts to adding an imaginary part to the mass since $r_{0}=2 \mathrm{M}$.

The prescription outlined above has its origin and basis in the method of complex paths which is outlined in Ref. [4 (see also [5]). This method is used to compute the transmission and reflection coefficients in standard semiclassical quantum mechanics by specifying a suitable complex contour for a given tunneling scenario. This contour is chosen between two semiclassical regions (where the wave function can be approximated using the semiclassical ansatz with negligible error) such that the semiclassical approximation holds all along the contour. If singularities, which represent distinctive features of the system under consideration, are present in the quantum system and these lie between the semiclassical regions, the appropriate complex contour contains useful information that decides the steady state behavior of the system. The complex path is identified from a region, say $L$ (a superposition of the incident and the reflected wave), to a second region $R$ (just contains the transmitted wave) is identified from $R$ to $L$ such that (a) all along the path the semiclassical ansatz is valid and (b) the reflected wave is exponentially greater than the incident wave atleast in the latter part of tha path near the region $L$. The transmitted wave is then moved along the path to obtain the reflected wave and thus the amplitude of reflection is identified in terms of the transmission amplitude. Having done this, the normalization condition is used, i.e., the sum of the modulus square of the transmission and reflection amplitudes should equal unity, to determine the exact values of the transmission and reflection coefficients.

In the black hole space-times considered in this section, the singularity that appears in the action functional in Eq. (10) is directly attributable to the presence of a horizon. Since the semiclassical approximation is applicable on either side of the horizon and arbitrarily close to it, the complex contours needed to bypass the singularity follow from the demand that the semiclassical approximation hold all along the contour. The type of singularity encountered here is similar to that encountered in the one-dimensional Schrödinger system with a potential of the form $\left(-1 / x^{2}\right)$. The method of complex paths gives the appropriate contours when dealing with right moving or left moving waves propagating across the singularity at $x=0$.

Consider therefore, an outgoing particle $\left(\partial S_{0} / \partial r>0\right)$ at $r=r_{1}<r_{0}$. The modulus square of the amplitude for this particle to cross the horizon gives the probability of emission of the particle. The contribution to $S_{0}$ in the ranges $\left(r_{1}, r_{0}-\epsilon\right)$ 
and $\left(r_{0}+\epsilon, r_{2}\right)$ is real. Therefore, choosing the contour to lie in the upper complex plane,

$$
\begin{aligned}
S_{0}[\text { emission }] & =-E \lim _{\epsilon \rightarrow 0} \int_{r_{0}-\epsilon}^{r_{0}+\epsilon} \frac{d r}{B(r)}+\text { (real part) } \\
& =\frac{i \pi E}{R\left(r_{0}\right)}+(\text { real part })
\end{aligned}
$$

where the minus sign in front of the integral corresponds to the initial condition that $\partial S_{0} / \partial r>0$ at $r=r_{1}<r_{0}$. For the sake of definiteness we have assumed $R\left(r_{0}\right)$ in Eq. (5) to be positive, so that $B(r)<0$ when $r<r_{0}$. (For the case when $R<0$, the answer has to be modified by a sign change.) The same result is obtained when an in-going particle $\left(\partial S_{0} / \partial r<0\right)$ is considered at $r=r_{1}<r_{0}$. The contour for this case must be chosen to lie in the lower complex plane. The amplitude for this particle to cross the horizon is the same as that of the outgoing particle due to the time reversal invariance symmetry obeyed by the system.

For an in-going particle $\left(\partial S_{0} / \partial r<0\right)$ at $r=r_{2}>r_{0}$, choosing the contour to lie in the upper complex plane, we get,

$$
S_{0}[\text { absorption }]=-\frac{i \pi E}{R\left(r_{0}\right)}+(\text { real part })
$$

The same result is obtained when an outgoing particle $\left(\partial S_{0} / \partial r>0\right)$ is considered at $r=r_{2}>r_{0}$. The contour for this case should be in the lower complex plane and the amplitude for this particle to cross the horizon is the same as that of the in-going particle due to time reversal invariance.

Taking the modulus square to obtain the probability $P$, we get,

$$
P[\text { emission }]=\exp \left(-\frac{4 \pi E}{\hbar R}\right) P \text { [absorption]. }
$$

Using the interpretation of Hartle and Hawking [3], the above relation shows the thermal behavior of emitted particles. Comparing Eq. (11) and Eq. (14), we identify the temperature of the horizon in terms of $R\left(r_{0}\right)$. Eq. (14) is based on the assumption that $R>0$. If $R<0$, one can show that, there will be a change of sign in the equation. Incorporating both the cases, the general formula for the horizon temperature $\beta^{-1}=\hbar|R| / 4 \pi$.

For the Schwarzschild black hole, $R=(2 M)^{-1}$, and the temperature is $\beta^{-1}=$ $\hbar / 8 \pi M$. For the de Sitter space-time, $R=H$, giving $\beta^{-1}=\hbar H / 2 \pi$. Although Reissner-Nordstrom space-time has a complicated space-time structure, using the method of complex paths we can obtain the correct temperature in a straight forward manner. The prescription given for handling the singularity is analogous to the analytic continuation in time proposed by Hartle and Hawking [3] to derive Black hole radiance.

\section{Non-singular coordinates in Schwarzschild manifold}

In this section, we discuss the properties of the two coordinates, Painlevé and Lemaitre, which are of our interest in this paper. The space-time structure of these two coordinates are best analyzed using $\mathrm{R}$ and $\mathrm{T}$ regions introduced by Novikov [16]. For the sake of completeness, we briefly outline key features of $\mathrm{R}$ and $\mathrm{T}$ regions of spherically symmetric space-times. 


\section{1. $R$ and $T$ regions for spherically symmetric space-times}

The general expression for the interval in a spherically symmetric field can be written in the following form:

$$
d s^{2}=e^{\nu}\left(d x^{0}\right)^{2}-e^{\lambda}\left(d x^{1}\right)^{2}-e^{\mu} d \Omega^{2} .
$$

Here $x^{0}$ is the time coordinate, $x^{1}$ is the radial space coordinate, and $d \Omega^{2}$ is the angular line element. [ $\nu, \lambda$ and $\mu$ are the functions of $x^{0}$ and $x^{1}$.] The reason for such a general line element for centrally symmetric gravitational field because the choice of "radius vector" is arbitrary. This is due to the fact that there is no quantity which has all the properties of the Euclidean radius vector. Hence, because of this arbitrariness in the choice of reference in general relativity, one can subject the coordinates to any transformation which does not destroy the spherical symmetry of the metric.

We assume that, at spatial infinity, the metric is Euclidean, and the expression for the interval in spherical coordinates becomes

$$
\left.d s^{2}\right|_{\infty}=\left(d x^{0}\right)^{2}-\left(d x^{1}\right)^{2}-\left(x^{1}\right)^{2} d \Omega^{2} .
$$

Changes of the coordinates $x^{0}$ and $x^{1}$

$$
x^{0}=x^{0}\left(x^{0^{\prime}}, x^{1^{\prime}}\right), \quad x^{1}=x^{1}\left(x^{0^{\prime}}, x^{1^{\prime}}\right)
$$

leave the spherical symmetry unaffected. Using a transformation of this type, we set

$$
x^{1}=e^{\mu\left(x^{0^{\prime}}, x^{1^{\prime}}\right) / 2}
$$

where the old coordinates have primes; then we select $x^{0}=x^{0}\left(x^{0^{\prime}}, x^{1^{\prime}}\right)$ so that the metric component $g_{10}$ vanishes, which is always possible. The expression for the interval in Eq. (15) takes on the form

$$
d s^{2}=e^{\nu\left(x^{0}, x^{1}\right)}\left(d x^{0}\right)^{2}-e^{\lambda\left(x^{0}, x^{1}\right)}\left(d x^{1}\right)^{2}-\left(x^{1}\right)^{2} d \Omega^{2} .
$$

The transformation in (18), which brings the interval to the form in (19) can, however, lead to a singular metric. The transformation in (18) can also lead to line elements of the form in (19), in which the metric component $g_{00}$ is negative and $g_{11}$ is positive. In such cases, the quantity $\left(g_{11}\right)^{1 / 2} d x^{1}$ measures the proper time of a particle in this coordinate system implying that $x^{1}$ should be treated as the time coordinate. Hence, the expression for the interval will read

$$
d s^{2}=e^{\nu\left(x^{0}, x^{1}\right)}\left(d x^{0}\right)^{2}-e^{\lambda\left(x^{0}, x^{1}\right)}\left(d x^{1}\right)^{2}-\left(x^{0}\right)^{2} d \Omega^{2} .
$$

Thus, by using a transformation of the type given in (18), one can bring the interval of a spherical gravitational field either to the form in (19) or to the form in (20). If at the given event in the general coordinate system in (15), the inequality,

$$
e^{\nu-\lambda}>\left(\frac{\partial \mu}{\partial x^{0}} / \frac{\partial \mu}{\partial x^{1}}\right)^{2}
$$

is satisfied, then the event is in an R-region. If the opposite inequality is satisfied, the event is in a T-region. The equality condition is an additional condition to the system of the gravitational field equations and therefore for an arbitrary spherically symmetric distribution in vacuum, it may not be possible to fulfill it in any four-dimensional region. The equality determines the boundary between $\mathrm{R}$ - and $\mathrm{T}$ - regions.

The definitions of R- and T- regions can be shown to be coordinate invariant. These will be found useful in understanding global space-time structure of Lemaitre and Painlevé coordinates. 


\subsection{The Lemaitre coordinate system}

We consider here the Lemaitre coordinate system which is a set of non-static coordinates like the Kruskal coordinate. The coordinate transformation from the Schwarzschild coordinates $(t, r)$ to Lemaitre coordinates are,

$$
\begin{aligned}
& \tau= \pm t \pm \sqrt{2 M} \int \frac{\sqrt{r} d r}{r-2 M}= \pm t \pm 2 M\left\{\ln \left(\frac{1-\sqrt{r / 2 M}}{1+\sqrt{r / 2 M}}\right)+2 \sqrt{\frac{r}{2 M}}\right\} \\
& R=t+\frac{1}{\sqrt{2 M}} \int \frac{r \sqrt{r} d r}{r-2 M}=t+2 M\left\{\ln \left[\frac{1-\sqrt{r / 2 M}}{1+\sqrt{r / 2 M}}\right]+2 \sqrt{\frac{r}{2 M}}\left[1+\frac{r}{6 M}\right]\right\}
\end{aligned}
$$

Choosing the positive sign in the above transformations, the Schwarzschild line element becomes,

$$
d s^{2}=d \tau^{2}-\frac{d R^{2}}{\left[\frac{3}{4 M}(R-\tau)\right]^{2 / 3}}-(2 M)^{2}\left[\frac{3}{4 M}(R-\tau)\right]^{4 / 3}\left(d \theta^{2}+\sin ^{2} \theta d \phi^{2}\right) .
$$

It is easy to see from the transformations in Eq. (22) that the Schwarzschild $r$ coordinate is given by the relation

$$
\left(\frac{r}{2 M}\right)=\left[\frac{3}{4 M}(R-\tau)\right]^{2 / 3}
$$

Hence the horizon $r=2 M$ corresponds to the value $3(R-\tau) / 4 M=1$. Note that the quantity $(R-\tau)$ can never be negative since it corresponds to the Schwarzschild radial coordinate. The coordinate $R$ is everywhere space-like while the coordinate $\tau$ is everywhere time-like. The metric above is non-stationary and test particles at rest relative to the reference system are particles moving freely in the given field. It can be easily shown (see Ref. [8]) that even in this coordinate, particles cannot remain at rest for $r<2 M$.

If we choose minus sign in Eq. (22), the Schwarzschild line element becomes

$$
d s^{2}=d \tau^{2}-\frac{d R^{2}}{\left[\frac{3}{4 M}(R+\tau)\right]^{2 / 3}}-(2 M)^{2}\left[\frac{3}{4 M}(R+\tau)\right]^{4 / 3}\left(d \theta^{2}+\sin ^{2} \theta d \phi^{2}\right) .
$$

Note that the sign of $\tau$ has changed. This metric describes a system where particles trajectories move outward from the singularity at $r=0$. This is analogous to the part of Kruskal coordinate where the past singularity and the time reversed Schwarzschild sector represent such a feature. This metric will be used when the action for outward moving particles needs to be considered. These coordinates can be modeled as that natural to a freely falling observer whose velocity at radial infinity is zero. The time coordinate $\tau$ measures the proper time of free falling observers; each observer moves along a line $R$ = constant. Figure (1) shows the Penrose diagram for the Schwarzschild geometry. The line-element (23) covers $\left(R, T_{-}\right)$and the line-element (25) covers $\left(R, T_{+}\right)$.

Using the criteria for R- and T- regions, defined in Sec. (3.1), we find that the region outside the Schwarzschild sphere, for the line element in (23), i.e., where

$$
\frac{3}{2}(R-\tau)>2 M
$$

is a R-region; but the region inside the Schwarzschild sphere, where the inequality is opposite to the above inequality, is a T-region. 


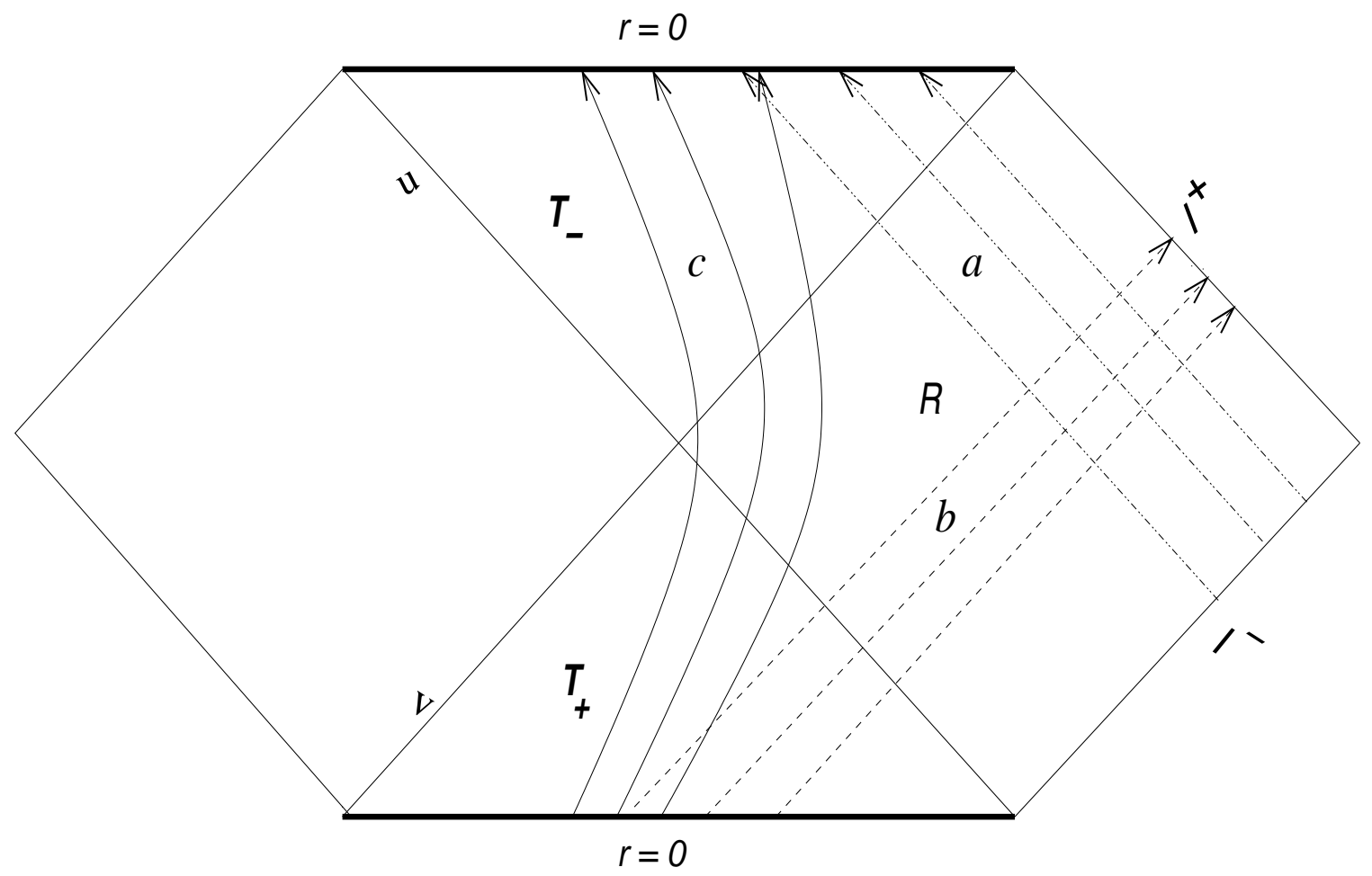

Figure 1. The Penrose diagram of the Schwarzschild space-time. $u$ and $v$ are the lines $r=2 M$; they are the boundaries of the $R$ and $T$ regions. $\left(R, T_{-}\right)$region correspopds to the line-element (23) while $\left(R, T_{+}\right)$region corresponds to that of (25). Lines $a, b$ and $c$ are the world lines of freely falling particles. $a$ are the geodesics of particles falling inward from $r=\infty$; $b$ are the geodesics of particles flying outward form $r=0$ to $r \rightarrow \infty$; and $c$ are the trajectories of the particles which move outward which eventually falls into the singularity.

In the T-region there is an obvious asymmetry in the direction of time flow. In the T-region $\left(\mathrm{T}_{-}\right)$, corresponding to the line element in (23), the test particles cannot remain at rest and these test particles will be directed towards $r=0$ where the curvature invariants are infinite. The line element in (25) will define second type of T-regions - an expanding region $\mathrm{T}_{+}$, which has completely opposite properties. Here all bodies move away from the singularity to the R-regions. A vacuum $\mathrm{T}_{+}$-region can be joined to the internal solution for an expanding sphere, but not to that for a contracting sphere as in the case for a $\mathrm{T}_{-}$-region. For a physically realizable event, the coexistence of $T_{+}$and $T_{-}$regions is impossible. In other words, the $\mathrm{T}$ region of the standard Schwarzschild is a doubly mapped $\mathrm{T}$ region in Lemaitre.

The above analysis will be useful for our semiclassical treatment given in Sec. (4.1). As mentioned earlier, the complex paths we use in our analysis, takes into account of all possible paths satisfying the semiclassical ansatz - irrespective of the multiple mapping of the part of the space-time. In principle, if the whole of space-time is doubly mapped w.r.t. the Schwarzschild space-time, there will be two distinct Rand T- regions. The complex paths, as used in Sec. (2), will have contributions from both of these which have no common point due to each path being in different R- and 
T- regions. Hence, the contribution to the amplitude of emission/absorption by these two paths will be mutually exclusive. In the case of Lemaitre coordinate, however, only part of the region(T-) is doubly mapped, it is always possible to find one point that is common to the paths contributing to absorption/emission. Hence, these paths are not mutually exclusive. These paths, on the other hand, will be mutually exclusive when one considers the probability amplitude - which is the important quantity in our approach. Hence, the action we obtain by regularizing the singularity and the resulting probability amplitude, for absorption/emission, will have equal contributions from both these paths.

\subsection{Painlevé metric}

In this section, we consider Painlevé coordinates, the other representation of the Schwarzschild space-time which is of our interest (for a discussion see Ref. [17]). The metric in these coordinates is non-singular at the horizon just as in the previous case but, unlike the Lemaitre metric, it is stationary. The coordinate transformation from the Schwarzschild coordinates $(t, r, \theta, \phi)$ to the Painlevé coordinates $\left(\tau_{P}, r, \theta, \phi\right)$ is given by

$$
\tau_{P}=t+2 \sqrt{2 M r}+2 M \ln \left(\frac{\sqrt{r}-\sqrt{2 M}}{\sqrt{r}+\sqrt{2 M}}\right) .
$$

Note that only the Schwarzschild time coordinate is transformed. The radial coordinate remains the same (as do the angular coordinates). The line element, in these new coordinates, is given by

$$
d s^{2}=\left(1-\frac{2 M}{r}\right) d \tau_{P}^{2}-2 \sqrt{\frac{2 M}{r}} d \tau_{P} d r-d r^{2}-r^{2} d \Omega^{2} .
$$

The metric is stationary and the constant time slice is simply flat Euclidean space. Another line element is easily obtained by considering the transformation of the form

$$
\tau_{P}=t-2 \sqrt{2 M r}-2 M \ln \left(\frac{\sqrt{r}-\sqrt{2 M}}{\sqrt{r}+\sqrt{2 M}}\right) .
$$

which leads to the equivalent line element

$$
d s^{2}=\left(1-\frac{2 M}{r}\right) d \tau_{P}^{2}+2 \sqrt{\frac{2 M}{r}} d \tau_{P} d r-d r^{2}-r^{2} d \Omega^{2}
$$

The line elements (28) and (30) can be written in a compact form

$$
d s^{2}=\left(d \tau_{P}\right)^{2}-\left(d r \pm \sqrt{\frac{2 M}{r}} d \tau_{P}\right)^{2}-r^{2} d \Omega^{2} .
$$

One can notice that the indefiniteness in the sign in coordinate transformation is same as encountered in Lemaitre coordinate. The line elements in Eq. (31) correspond to the ingoing and outgoing null geodesics. [Figure (2) shows the Penrose diagram of the Schwarzschild space. As described earlier, $\left(r, \tau_{P}\right)$ for the upper sign in the above line-element cover regions $R$ and $T$.] In terms of $r$ and $\tau_{P}$, these are given by

$$
\begin{aligned}
& \text { ingoing : } \tau_{P}+r-2 \sqrt{2 M r}+4 M \log [\sqrt{r}+\sqrt{2 M}]=v=\text { constant } \\
& \text { outgoing : } \tau_{P}-r-2 \sqrt{2 M r}-4 M \log [\sqrt{r}+\sqrt{2 M}]=u=\text { constant }
\end{aligned}
$$




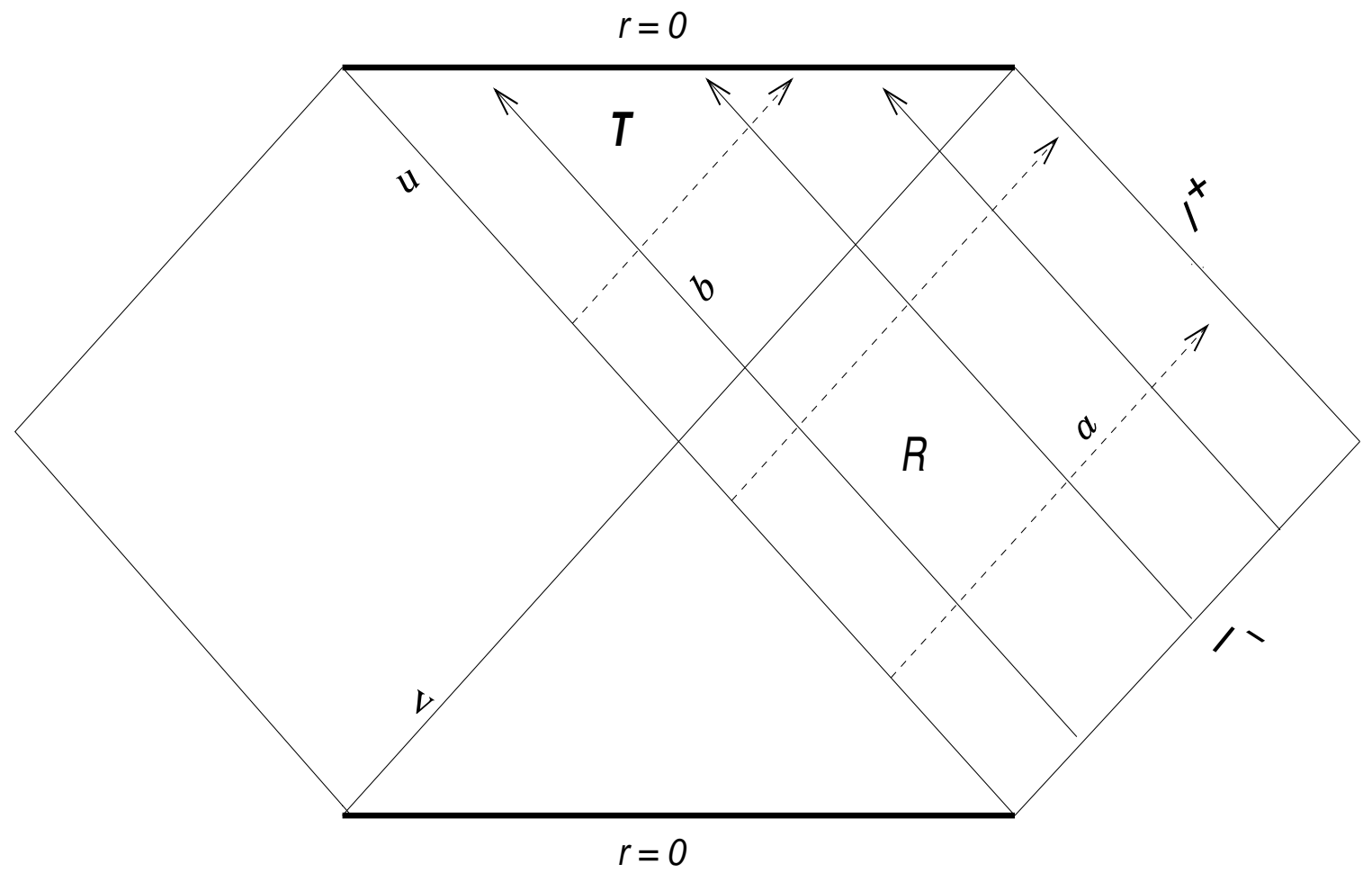

Figure 2. The Penrose diagram of the Schwarzschild space-time. $u$ and $v$ are the lines $r=2 M$. $(R, T)$ region corresponds to the line-element (28). Lines $a, b$ are the light rays. $a$ are the outgoing light rays and these cannot be traced back past the horizon $u ; b$ are the ingoing light rays and these fall into the singularity

In the case of Lemaitre coordinate, we identified that the physical extension of the external Schwarzschild space-time to the region inside the Schwarzschild sphere has a doubled valued nature by splitting the space-time defined using $\mathrm{R}$ - and $\mathrm{T}$ - regions. We make use of the same formalism here to analyze the space-time structure of the Painlevé metric.

In our earlier discussion of R- and T- regions in Sec. (3.1), these regions were defined for the line elements not involving mixed components $g_{0 i}$. The mixed components, for spherically symmetric space-times, can be set equal to zero inasmuch as rotation is absent by the assumption of sphericity. Hence in the literature, the definition of $\mathrm{R}$ - and $\mathrm{T}$ - regions for space-times with the mixed components is not considered. In order to identify $\mathrm{R}$ - and $\mathrm{T}$ - regions in the Painlevé coordinate, we first obtain below a transformation connecting Painlevé and Lemaitre coordinates $(\tau, R, \theta, \phi)$.

Noting that the constant time slice is a flat Euclidean space for the Painlevé metric, we transform the coordinate variables of Lemaitre as

$$
\tau_{P}=\tau, \quad r=(2 M)^{1 / 3}\left(\frac{3}{2}(R-\tau)\right)^{2 / 3}
$$

to go from Lemaitre to Painlevé line elements. Under these transformations, the Lemaitre line element in (23) now translates into Painlevé line element in (28). The 
other Painlevé line element in (30) is obtained by repeating the same steps by starting from the Lemaitre coordinate in (25), which is the time reversal of the line element in (23).

The criterion of R- and T- regions for Painlevé metric can be easily obtained from Eq. (26). For the line elements in (28) and (30), the inequality satisfied for the Rregion is $r>2 M$. Hence, for the two line elements of the Painlevé metric, $\mathrm{R}$ - and Tregions are the same - the whole of the space-time is doubly mapped. As mentioned in the earlier subsection, the contribution to the amplitude of absorption/emission will be from two mutually exclusive paths.

\section{Semiclassical treatment}

In this section, we consider particle production in the two coordinate systems described in the previous section by the semiclassical method which was outlined in Sec. (2). The semiclassical wavefunction for the quantized scalar field and consequently the semiclassical propagator is written down and by regularizing the singularity in the action functional by complex extension and Hawking radiation is obtained for both these coordinate systems. Before, we proceed with the application of the method of complex paths in these coordinates, we would like to point the readers that the method of complex paths does not require the Killing vectors to be time-like. Noting that the method requires to separate the non-linear partial differential equation into an ordinary differential equation for identifying the singularities, we need to use the ansatz,

$$
S=-E \xi+f(\rho)
$$

where $\xi$ represents the coordinate associted to the Killing vector. However, the Killing vector need not coincide asymptotically with the Minkowski time. In the case of Painlevé (and Schwarzschild), as we will notice, $\xi$ coincides with the Minkowski time asymptotically while $\xi$ is a light cone coordinate in the case of Lemaitre and does not coincide with the Minkowski coordinate asymptotically.

\subsection{Lemaitre coordinate}

The method described in Sec. (2) in obtaining the thermal spectrum for the Schwarzschild coordinate will be used here without modification. We consider the minimally, coupled, massless, scalar field propagating in the Lemaitre background. The system we first consider is that for in-going particles described by the metric in Eq. (23). Since the entire physics is contained in the $(\tau, R)$ plane, we separate out the angular variables and set $\Phi=\Psi(\tau, R) Y_{l}^{m}(\theta, \phi)$. The equation satisfied by $\Psi$ is

$$
\begin{aligned}
& {\left[1-U^{2 / 3}\right]\left(\partial_{U}^{2}+\partial_{V}^{2}\right) \Psi-2\left[1+U^{2 / 3}\right] \partial_{U} \partial_{V} \Psi } \\
+ & {\left[\frac{1}{U}-\frac{5 U^{-1 / 3}}{3}\right]\left(\partial_{U}^{2}-\partial_{V}^{2}\right) \Psi=\frac{16 l(l+1)}{9 U^{4 / 3}} \Psi }
\end{aligned}
$$

where $U$ and $V$ are dimensionless parameters given by

$$
U=\frac{3}{4 M}(R-\tau), \quad V=\frac{3}{4 M}(R+\tau) .
$$


Note that the horizon is the surface $U=1$. Making the usual semiclassical ansatz for $\Psi$ as done in Eq. (7) and expanding the $S$ in powers of $\hbar$ as done in Eq. (8) one finds, to lowest order,

$$
\left(1-U^{2 / 3}\right)\left[\left(\partial_{U} S_{0}\right)^{2}+\left(\partial_{V} S_{0}\right)^{2}\right]-2\left(1+U^{2 / 3}\right)\left(\partial_{U} S_{0}\right)\left(\partial_{V} S_{0}\right)+\frac{16 l(l+1)}{9 U^{2 / 3}}=0 .
$$

The above equation is just the Hamilton-Jacobi equation satisfied by a massless particle moving in the space time determined by the line element in (23). Specializing to the case $l=0$ for simplicity, and making the ansatz $S_{0}=-4 M E V / 3+f(U)$, we obtain,

$$
\frac{d f}{d U}=E \frac{1+U^{2 / 3} \pm 2 U^{1 / 3}}{1-U^{2 / 3}},
$$

where the \pm signs arise from taking square roots. Notice that the denominator is singular at $U=1$ only for the positive sign. This singular solution for $f$ evidently corresponds to outgoing particles $\left[\left(\partial S_{0} / \partial U\right)>0\right]$. Therefore choosing the positive sign and making the convenient change of variable $x^{3}=U$, the solution to Eq. (37) is given by

$$
S_{0}[\text { outgoing }]=-\frac{4 M E}{3} V+4 M E \int d x \frac{x^{2}(1+x)}{1-x} .
$$

[Note: The constant of integration $E$ is the "energy" of the particle corresponding to the dimensionless variable $V$. In order to get the correct definition (of $E$ at the future infinity), we need to set $S=-4 M E V / 3+f(U)$.] It is clear that the action function is singular at the horizon $x=1$. Following the method outlined in Sec. (2) and regularizing the singularity by complex contour lying in the upper complex plane, we obtain

$$
S_{0}[\text { emission }]=\text { real part }+8 i \pi M E
$$

In order to obtain the action for absorption of particles corresponding to the ingoing particles $\left[\left(\partial S_{0} / \partial x\right)<0\right]$, we have to repeat the above calculation using the metric given in Eq. (25). In this case, it is easy to see that the only singular solution corresponds to in-going particles and so

$$
S_{0}[\text { absorption }]=\text { real part }-8 i \pi M E
$$

where the minus sign arises from choosing the appropriate complex contour given by the semiclassical prescription outlined in Sec. (2). [Note here that in the calculation of $S_{0}$, we do not require the prescription of pushing the singularity at the horizon required in the case of Schwarzschild coordinate in Ref. [4].] Constructing the semiclassical propagator in the usual manner and taking the modulus square we obtain the probability. From the earlier discussions [see Sec. (3.3)], we know that in calculating the probability of absorption/emission there is an extra contribution due to multiple mapping of part of the space-time and that the total contribution to the probability is from four sets of the complex paths satisfying the semiclassical ansatz. Taking this into account by dividing $S_{0}$ by four, we obtain

$$
P[\text { emission }]=\exp (-8 \pi M E) P[\text { absorption }] .
$$

Thus, the exponential dependence of the energy allows us to give the thermal interpretation to this result, which shows that the temperature of the emission spectrum is the standard Hawking temperature. [Note that the if we translate the action in Eq. (40) in terms of the Schwarzschild coordinates (t,r), we find 
that $S_{0}[$ emission $]=-2 E t+F(r)$. The factor 2 appears in the action due to the multiple mapping of the part of the Lemaitre coordinate (w. r. t. the Schwarzschild coordinate).]

In Ref.[何, the authors have shown explicitly that close to the horizon, the terms containing the mass and angular part does not contribute significantly. Similar results hold in our case and the above analysis is therefore applicable to both massless and massive scalar particles.

It has been shown by Davies 18] that a freely falling detector will see a particle spectrum different from the thermal spectrum. Comparison with our result shows that there is no correspondence between the particles detected by the model detector and the particle spectrum obtained by the field theoretic analysis - a result well known in other contexts as well.

In the next subsection, we will do similar analysis for the Painlevé metric and obtain the Hawking temperature by regularizing the singularity in the action functional by the complex extension.

\subsection{Painlevé metric}

We proceed with the semiclassical analysis for the Painlevé metric, as done in the previous subsection. As before, we consider a minimally coupled massless scalar field $\Phi$. Since all the relevant physics is contained in the $\left(\tau_{P}, r\right)$ plane, we set $\Phi=\Psi\left(\tau_{P}, r\right) Y_{l}^{m}(\theta, \phi)$ beforehand. The equation satisfied by $\Psi$ is,

$$
\begin{aligned}
& \frac{\partial^{2} \Psi}{\partial \tau_{P}^{2}}-2 \sqrt{\frac{2 M}{r}} \frac{\partial^{2} \Psi}{\partial \tau_{P} \partial r}-\frac{3}{2 r} \sqrt{\frac{2 M}{r}} \frac{\partial \Psi}{\partial \tau_{P}} \\
- & \left(1-\frac{2 M}{r}\right) \frac{\partial^{2} \Psi}{\partial r^{2}}-\frac{2}{r}\left(1-\frac{M}{r}\right) \frac{\partial \Psi}{\partial r}-\frac{l(l+1)}{r^{2}} \Psi=0 .
\end{aligned}
$$

Making the usual semiclassical ansatz for $\Psi$ as done in Eq. (7) and expanding the $S$ in powers of $\hbar$ as done in Eq. (8) one finds, to lowest order,

$$
-\left(\frac{\partial S_{0}}{\partial \tau_{P}}\right)^{2}+2 \sqrt{\frac{2 M}{r}}\left(\frac{\partial S_{0}}{\partial \tau_{P}}\right)\left(\frac{\partial S_{0}}{\partial r}\right)+\left(1-\frac{2 M}{r}\right)\left(\frac{\partial S_{0}}{\partial r}\right)^{2}+\frac{l(l+1) \hbar^{2}}{r^{2}}=0 .
$$

This is the Hamilton-Jacobi equation for a massless particle moving in a space- time determined by the metric Eq. (28). Introducing a dimensionless variable $\rho=r / 2 M$ and considering the s-waves, i.e. $l=0$, for simplicity, the solution to the above equation is easily found to be,

$$
S_{0}=-E \tau_{P}+2 M E \int d \rho \frac{\sqrt{\rho}(1 \pm \sqrt{\rho})}{\rho-1} .
$$

The denominator is singular at $\rho=1$ only for the positive sign. Note that $\rho=1$ corresponds to the black hole horizon. The singular solution for $S_{0}$ evidently corresponds to outgoing particles $\left[\left(\partial S_{0} / \partial \rho\right)>0\right]$. Following the method outlined in Sec. (2) and regularizing the singularity by a complex contour lying in the upper complex plane and using the arguments in Sec. (3.3), we obtain

$$
S_{0}[\text { emission }]=\text { real part }+2 i \pi M E \text {. }
$$

In this case, the action that has been calculated is interpreted to be that for emission by analogy with that done in Sec. (2). In order to obtain the action for absorption of particles corresponding to the ingoing particles $\left[\left(\partial S_{0} / \partial \rho\right)<0\right]$, we have to consider 
the Hamilton-Jacobi equation for the metric in Eq. (30) and repeating the above calculations and using the arguments above we obtain

$$
S_{0}[\text { absorption }]=\text { real part }-2 i \pi M E,
$$

where the minus sign arises from choosing the complex contour lying in the upper complex plane given by the semiclassical prescription outlined in Sec. (2). Here again we notice that the calculation of $S_{0}$ does not require any prescription, just like in the Lemaitre coordinate, as against the Schwarzschild coordinate. By constructing the semiclassical propagator in the usual manner and taking the modulus square to get the probability, we obtain

$$
P[\text { emission }]=\exp (-8 \pi M E) P[\text { absorption }] .
$$

Here again, the exponential dependence of the energy allows us to give the thermal interpretation to this result, which shows that the temperature of the emission spectrum is the standard Hawking temperature. It may be noted that in our interpretation, we consider the amplitude for pair creation both inside and outside the horizon. This is different from the treatment of Hawking radiation obtained recently by Parikh and Wilczek [9]. The authors considered Hawking radiation as a pair creation outside the horizon, with the negative energy particle tunneling into the black hole. The tunneling of particles produced just inside the horizon also contributes to the Hawking radiation and is incorporated in our formalism.

\section{Conclusions}

We have studied the spectrum of created particles in two coordinate representations, Painlevé and Lemaitre, of the Schwarzschild space-time for a linear, massless scalar field using the method of complex paths. We have found that in both these coordinate representations the spectrum of radiation is thermal and the temperature is same as the standard Hawking temperature.

The classical space-time analysis of these two coordinates is done using R- and T- regions introduced by Novikov [16]. In Ref. [3], Hartle and Hawking calculated the probability amplitude for the emission and absorption by identifying the particular path for the particles to cross the horizon, i.e. the in-going and out-going paths which requires a detailed study of particle trajectories. As we have seen, analysis of these two coordinates, Painlevé and Lemaitre, using R- and T- regions provides an elegant method in understanding the global structure of the space-time, thus making the detailed analysis of particle trajectories unnecessary.

The physical effect of Hawking radiation is related to the decrease in the mass of the black hole; the mass being converted into energy, thus implying the radiation emitted should be covariant. In order to prove the covariance of Hawking radiation, we would like to have a general proof for all the coordinate representations of the Schwarzschild space-time. However, a general result showing the 'general covariance of Hawking radiation' is really a difficult task. Hence, we have considered two coordinate systems whose properties are different from that of the Schwarzschild metric i.e. both are non-static and do not possess singularity at the horizon. Using the method of complex paths, we have shown that in both these coordinate representations, Painlevé and Lemaitre, the spectrum of particle emitted is thermal and the temperature is the standard Hawking temperature $(1 /(8 \pi M))$ and hence indicating covariance of the Hawking radiation as far as these coordinates are concerned. Thus, we have shown 
that even-though the action satisfies a generally covariant HJ equation, when one transforms coordinates, the action integral develops poles and this can be interpreted by using Novikov's $R$ and $T$ regions analysis of the space-time manifold.

The analysis which we have performed, in our paper, considers the amplitude for pair creation for both inside and outside the horizon. Our treatment is different from the treatment of Hawking radiation obtained by Parikh and Wilczek [9]. In our approach, the tunneling of particles just produced inside the horizon (tunneling in the context of non-relativistic quantum mechanics)as well as the pair creation just outside the horizon (over the barrier reflection) contributes to the Hawking radiation.

The only analysis that has been performed in the literature is that of the particle spectrum of a freely falling detector. It has been shown by Davies 18 that a freely falling detector will see a particle spectrum different from the thermal spectrum. Comparison with results of Lemaitre coordinate, which is natural to a freely falling observer, shows that there is no correspondence between the particles detected by the model detector and the particle spectrum obtained by the field theoretic analysis - a result known in contexts of flat space-times (see Ref. [1] and references therein).

\section{Acknowledgments}

The authors would wish to thank Prof. M.A.H. MacCallum for providing with the English translation of Novikov's work before it's publication in GRG. S.S. is being supported by the Council of Scientific and Industrial Research, India.

\section{References}

[1] L. Sriramkumar and T. Padmanabhan, Probes of the vacuum structure of quantum fields in classical backgrounds, Int. J. Mod. Phys. D 111 (2002); gr-qc/9903054.

[2] S. Hawking, Commun. Math. Phys. 43, 199(1975).

[3] J.B. Hartle and S.W. Hawking, Phys. Rev. D 13, 2188 (1976).

[4] K. Srinivasan and T. Padmanabhan, Particle production and complex path analysis, Phys. Rev. D 60, 24007 (1999).

[5] L. D. Landau and E. M. Lifshitz, Quantum Mechanics (Non-relativistic Theory), Course of Theoretical Physics, Volume 3 (Pergamon Press, New York, 1975).

[6] P. Candelas, Vacuum polarization in Schwarzschild spacetime, Phys. Rev. D 21, 2185 (1980).

[7] M. Visser, Gravitational vacuum polarization: I, II, III, Phys. Rev. D 54, 5103 - 5128 (1996).

[8] L. D. Landau and E. M. Lifshitz, Classical Theory of Fields, Course of Theoretical Physics, Volume 2 (Pergamon Press, New York, 1975).

[9] M. K. Parikh and F. Wilczek, Hawking Radiation as Tunneling, Phys. Rev. Lett. 85, 5042 (2000); hep-th/9907001.

[10] R. Schutzhold, On the Hawking effect, Phys. Rev. D 64, 024029 (2001); gr-qc/0011047

[11] W. G. Unruh, Phys. Rev. Lett. 46, 1351 (1981).

[12] T. Jacobson, Phys. Rev. D 44, 1731 (1991); W. G. Unruh, ibid 51, 2827 (1995); S. Corley and T. Jacobson, ibid 59, 124011 (1999).

[13] T. Padmanabhan, Pramana-J. Phys. 37, 179 (1991).

[14] T. Padmanabhan, Why does an accelerated detector click?, Class. Quan. Grav. 2, 117 (1985).

[15] N. D. Birrel and P. C. W. Davies, Quantum Field in Curved Space (Cambridge University Press, Cambridge, England, 1982).

[16] I. D. Novikov, Communications of the Shternberg state Astronomical Institute, 132, 3-42 (1964).

[17] P. Kraus and F. Wilczek, Some Applications of a Simple Stationary Line Element for the Schwarzschild Geometry, gr-qc/9406042.

[18] P.C.W. Davies, Rep. Prog. Phys. 41, 1315 (1976).

[19] J. R. Letaw, Phys. Rev. D. 23, 1709 (1981); J. R. Letaw and J. D. Pfautsch, ibid 24, 1491 (1981). 